\title{
Effect of different forms of static air-gap eccentricity on stator magnetic pull per unit area of turbo-generator
}

\author{
Weiqi Deng, Yuling He ${ }^{a}$, Guiji Tang, Yuchao Meng and Hao Zhong \\ Deparment of Mechanical Engineering, North China Electric Power University, Baoding, P.R. China
}

\begin{abstract}
This paper investigates the stator magnetic pull per unit area (MPPUA) and stator vibration characteristics under different static air-gap eccentricity (SAGE) forms of turbo-generator. Three types of SAGE, i.e. the rotor offset, the stator deformation, and the mixed one composed of the former two, are taken into account. Firstly, the MPPUA under different SAGE conditions are analyzed, based on which the formulas of the magnetic flux density (MFD) are deduced. Then the simulation study of a SDF-9 non-salient pole synchronous generator is taken with Ansoft Maxwell. It is shown that the SAGE will cause the stator vibration at $2 f$ ( $f$ is the electrical frequency). The vibration amplitudes under rotor offset are larger than those under stator deformation. As SAGE develops, the vibration amplitudes rotor offset will all be increased, especially the 2nd harmonic vibration, while the vibration basically unchanged under stator ellipse deformation. The achievements are beneficial to the SAGE fault identification.
\end{abstract}

Keywords: stator air-gap eccentricity (SAGE); magnetic pull per unit area (MPPUA); turbo-generator; simulation calculation; fault diagnosis.

\section{Introduction}

At present, the research on the unbalanced magnetic pull (UMP) and the vibration characteristics of turbo-generator under the static eccentricity fault focus on the rotor side. Many researchers use the vibration characteristics of the rotor to diagnose the faults of the generator [1-4]. Others focus on the electrical characteristic parameter. Ref.[5] analyzes the electromotive force characteristic of stator armature under the inter-turn short circuit fault detection in permanent magnet synchronous machines (PMSM) to determine the degree and location of the fault. Ref.[6] using wavelet analysis on stator current to detect of doubly-fed induction generator inter-turn stator fault which commonly applies in wind farms. Ref.[7] provides a shaft voltage measurements to detect static eccentricity. Ref.[8] applies and expands the machine voltage signature analysis technique, it shows that a static eccentricity makes rise a double fundamental frequency ripple in the rotor current of salient-pole synchronous machines. However, due to the structure of the generator, the vibration data of the rotor can not be obtained directly, but through the bearing seat instead. This may cause the vibration data not accurate. In this paper, the stator vibration data, which can be directly obtained from stator core, are used to realize online monitoring and trouble diagnosing of the generator.

Besides, many researchers in the study of SAGE fault just considering the single rotor offset static

\footnotetext{
a Corresponding author : heyuling1@163.com
} 
eccentricity fault, which is not comprehensive. For example, stator core will be distorted when be heated, and lead to stator core deformation, which can be divided into local deformation and overall elliptical deformation. In this paper, the expressions of MPPUA will be deduced under the action of various static eccentricities. Moreover, the literature above did not involve fault experiments cause by stator core deformation or mixed static eccentricity, because it is hard to change the stator shape. In this article, taking the turbo generator as the research object, rotor static air-gap eccentricity, stator static air-gap eccentricity and mixed static eccentricity fault models are built on the basis of Ansoft Maxwell, and the MPPUA and vibration characteristics cause by these radial static air-gap eccentricity are analyzed by simulation measurement.

\section{Theoretical analysis}

\subsection{Air-gap magnetic density analysis}

According to Ref. [2], the air-gap magnetomotive force (MMF) in normal condition can be written as

$$
\begin{gathered}
f\left(\alpha_{m}, t\right)=F_{r} \cos \left(\omega_{\mathrm{r}} t-\alpha_{m}\right)+F_{s} \cos \left(\omega_{\mathrm{r}} t-\alpha_{m}-\psi-\frac{\pi}{2}\right)=F_{1} \cos \left(\omega_{\mathrm{r}} t-\alpha_{m}-\beta\right) \\
\left\{\begin{array}{l}
F_{1}=\sqrt{F_{s}^{2} \cos ^{2} \psi+\left(F_{r}-F_{s} \sin \psi\right)^{2}} \\
\beta=\operatorname{arctg} \frac{F_{s} \cos \psi}{F_{r}-F_{s} \sin \psi}
\end{array}\right.
\end{gathered}
$$

where $F_{r}, F_{s}$ and $F_{1}$ are respectively the rotor MMF, the stator MMF, and the composite MMF at the fundamental frequency, $\omega_{r}=2 \pi f_{r}$ is the rotor mechanical angular frequency, $f_{r}$ is the rotating frequency of rotor (for turbo-generator, $f_{r}$ equals to the electrical frequency $f$, and hereafter, we write $f_{r}$ as $f$ and $\omega_{r}$ as $\omega$ for short), $\alpha_{m}$ is the mechanical angle to indicate the circumferential location of the air-gap, and $\psi$ is the internal power angle of the generator.

In this paper, we consider different forms of SAGE respectively caused by rotor offset, stator deformation, and the mixed condition of these two, as indicated in Fig.1. For the sake of universality, considering the possible SAGE cases together, the radial air-gap length can be written as

$$
g\left(\alpha_{m}\right)=g_{0}\left[1-\delta_{s} \cos \alpha_{m}-\sum \delta_{s n}^{\prime} \cos \left(\alpha_{m}+\theta_{n}\right)\right]
$$

where $g_{0}$ is the average value of the air-gap, $\delta_{s}$ is the relatively static eccentricity caused by rotor offset, $\delta_{s n}^{\prime}$ is the relatively static eccentricity caused by stator deformation ( $\delta_{s n}^{\prime}$ denotes to reduce the air-gap, while $-\delta_{s n}^{\prime}$ denotes to increase the air-gap), $n$ is the total number of the stator static eccentricities, $\theta_{n}$ is the angle between the $n$-th stator static eccentricity and $\mathrm{X}$ axis.

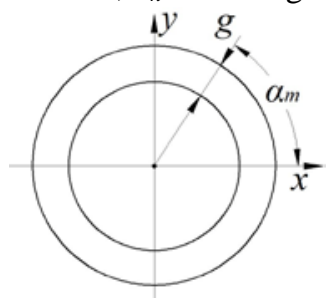

(a)

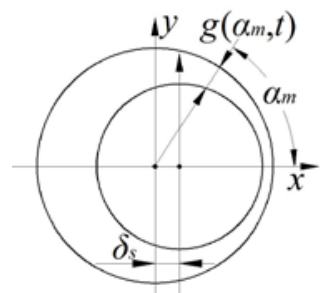

(b)

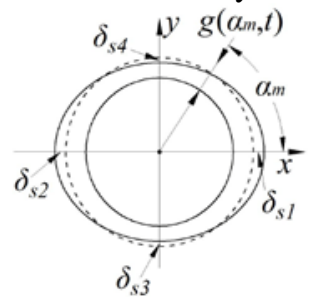

(c)

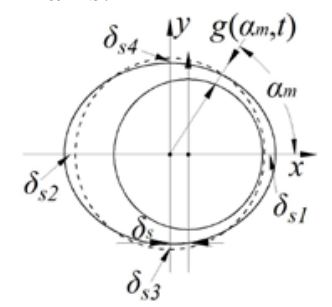

(d)

Figure 1. Different forms of SAGE in generator: (a) normal, (b) SAGE caused by rotor offset, (c) SAGE caused by stator core ellipse, and (d) mixed SAGE example caused by rotor offset and stator core ellipse

The air-gap permeance can be obtained by power series expansion, and it can be finally written as 


$$
\Lambda\left(\alpha_{m}\right)=1 / g\left(\alpha_{m}\right)=\Lambda_{0}+\Lambda_{s} \cos \alpha_{m}+\sum \Lambda_{s n}^{\prime} \cos \left(\alpha_{m}+\theta_{n}\right)
$$

where $\Lambda_{0}$ is the constant component of the air-gap permeance, $\Lambda_{s}=\Lambda_{0} \delta_{s}$ is the component of the permeance caused by rotor offset, and $\Lambda_{s n}^{\prime}=\Lambda_{0} \delta^{\prime}$ sn is the component caused by stator deformation.

Then the MFD in the air-gap is

$$
B\left(\alpha_{m}, t\right)=f\left(\alpha_{m}, t\right) \Lambda\left(\alpha_{m}\right)=F_{1} \cos \left(\omega_{\mathrm{r}} t-\alpha_{m}-\beta\right)\left[\Lambda_{0}+\Lambda_{s} \cos \alpha_{m}+\sum \Lambda_{s n}^{\prime} \cos \left(\alpha_{m}+\theta_{n}\right)\right]
$$

\subsection{Stator structure and MPPUA analysis}

Stator core is made up of a lot of fan-shaped steel sheets. The inner surface of the stator core can be excited by the action of exciting force when the generator is running. The external force, which is also called UMP, acting on the stator core will be zero while the MPPUA is not. Considering the shell structure of the stator core, which is piled up with si-iron plates, the stiffness of it can be small, so study the pulsating MPPUA is more significant than the external UMP.

MPPUA can be calculated by Eq.(6), which is widely used by scholars [1]

$$
q\left(\alpha_{m}, t\right)=B^{2}\left(\alpha_{m}, t\right) / 2 \mu_{0}
$$

where $\mu_{0}$ is the air permeability, and the stator MPPUA can be written as Eq.(7).

$$
\begin{aligned}
q(\alpha, t) & =\frac{F_{1}^{2} \Lambda_{0}^{2}}{2 \mu_{0}}\left\{\frac{1}{2}+\frac{1}{4} \delta_{s}^{2}+\delta_{s} \cos \alpha+\frac{1}{4} \delta_{s}^{2} \cos 2 \alpha+\frac{1}{2} \cos \left(2 \omega_{r} t-2 \alpha-2 \beta\right)+\frac{1}{2} \delta_{s} \cos \left(2 \omega_{r} t-\alpha-2 \beta\right)\right. \\
& +\frac{1}{2} \delta_{s} \cos \left(2 \omega_{r} t-3 \alpha-2 \beta\right)+\frac{1}{8} \delta_{s}^{2} \cos \left(2 \omega_{r} t-2 \beta\right)+\frac{1}{4} \delta_{s}^{2} \cos \left(2 \omega_{r} t-2 \alpha-2 \beta\right)+\frac{1}{8} \delta_{s}^{2} \cos \left(2 \omega_{r} t-4 \alpha-2 \beta\right) \\
& +\sum \delta_{s n}^{\prime} \cos \left(\theta_{n}-\alpha\right)+\frac{1}{2} \sum \delta_{s n}^{\prime} \cos \left(2 \omega_{r} t-\alpha-2 \beta-\theta_{n}\right)+\frac{1}{2} \sum \delta_{s n}^{\prime} \cos \left(2 \omega_{r} t-3 \alpha-2 \beta+\theta_{n}\right)+\frac{1}{2}\left[\sum \delta_{s n}^{\prime} \cos \left(\theta_{n}-\alpha\right)\right]^{2} \\
& +\frac{1}{2} \cos \left(2 \omega_{r} t-2 \alpha-2 \beta\right)\left[\sum \delta_{s n}^{\prime} \cos \left(\theta_{n}-\alpha\right)\right]^{2}+\frac{1}{2} \delta_{s} \sum \delta_{s n}^{\prime} \cos \theta_{n}+\frac{1}{2} \delta_{s} \sum \delta_{s n}^{\prime} \cos \left(\theta_{n}-2 \alpha\right)+\frac{1}{4} \delta_{s} \sum \delta_{s n}^{\prime} \cos \left(2 \omega_{r} t-2 \beta-\theta_{n}\right) \\
& \left.+\frac{1}{4} \delta_{s} \sum \delta_{s n}^{\prime} \cos \left(2 \omega_{r} t-2 \alpha-2 \beta+\theta_{n}\right)+\frac{1}{4} \delta_{s} \sum \delta_{s n}^{\prime} \cos \left(2 \omega_{r} t-4 \alpha-2 \beta+\theta_{n}\right)+\frac{1}{4} \delta_{s} \sum \delta_{s n}^{\prime} \cos \left(2 \omega_{r} t-2 \alpha-2 \beta-\theta_{n}\right)\right\}
\end{aligned}
$$

From Eq.(7), it can be seen that MPPUA consists of two parts: DC component and pulsating components at $2 f$. Moreover, the component amplitudes will vary due to different SAGE cases. Qualitatively, the DC component will not bring in vibrations but generate a deformation tendency to the stator after a long period action, while the pulsating force components will induce radial vibrations at the pulsating frequency, i.e. $2 f$.

\subsection{Vibration characteristics analysis}

In the case of rotor offset, the air-gap between rotor and stator become one side big and the other side is small, the MPPUA should be asymmetrical and it can be much bigger where the air-gap is small. Besides the air-gap is overall change, so the amplitude of MPPUA around the stator inner surface will all change. And as the SAGE develops, the amplitudes of DC component and $2 f$ component are both increasing.

Differently, in the case of stator deformation, the SAGE will cause the MPPUA vibration and the distribution of MPPUA is related to the deformation number $n$, the level of deformation $\Lambda_{s n}^{\prime}$, and the angle $\theta_{n}$ between the $n$-th deformation and X axis. In general, the MPPUA is negatively correlated with the size of the air-gap. And if the deformations are symmetrically distributed in preciseness, the integral MPPUA acting on the stator can be zero, and cause no vibration.

The mixed SAGE combines the characteristics of both rotor offset and stator deformation, which we can confirm from Eq.(7), the mixed SAGE is the superposition of rotor offset and stator deformation. The change of MPPUA is affected by the stator deformation numbers, positions and 
degrees, and the rotor offset positions and degrees at the meantime.

\section{Verification and analysis}

\subsection{Verification method and setting}

Taking SDF-9 type non-salient generator in the National Key Lab of New Energy and Electric Power System, North China Electric Power University, as simulation object, the primary parameters of the generator are shown in Tab.1. It is a fault simulating generator which can carry out the stator and rotor inter-turn short circuit faults and the rotor SAGE, see in Fig.2 (a). However, it can not simulate stator SAGE fault and mixed SAGE fault, therefore here it has to take a simulation work instead.

Simulation experiment using the finite element software Ansoft Maxwell 15, and the generator's model is shown in Fig.2 (c). The rated capacity of the generator is 7.5KVA. During the simulation, the exciting current is set to $0.4 \mathrm{~A}$ and the rotating speed is $3000 \mathrm{r} / \mathrm{min}$. Calculating the stator MPPUA characteristics under various conditions in Tab.2.

Table 1. Primary parameters of SDF-9 type generator

\begin{tabular}{|l|l|l|l|}
\hline parameters & values & parameters & values \\
\hline Rated capacity & $7.5 \mathrm{kVA}$ & Axial air-gap length & $l=100 \mathrm{~mm}$ \\
\hline Rated voltage & $400 \mathrm{~V}$ & Number of stator slots & $Z_{1}=24$ \\
\hline Power factor & $\cos \varphi=0.8$ & Ratio of pitch to polar distance & $k_{\mathrm{v}}=y / \tau=0.83$ \\
\hline Rated rotating speed & $n_{r}=3000 \mathrm{rpm}$ & pitch-shortening value & $k_{p}=0.966$ \\
\hline Number of pole-pairs & $p=1$ & distribution coefficient & $k_{d}=0.958$ \\
\hline Radial air-gap length & $g_{0}=0.8 \mathrm{~mm}$ & Number of parallel branches & $a=2$ \\
\hline
\end{tabular}

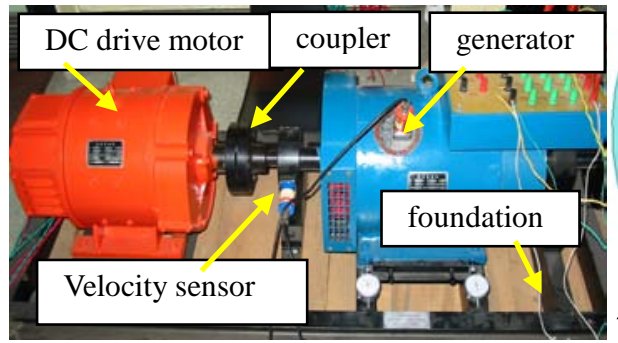

(a)

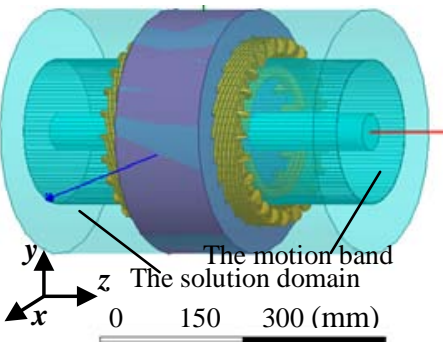

(b)

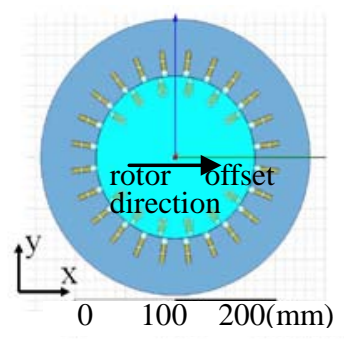

(c)

Figure 2. The study object: SDF-9 type fault simulating generator: (a) Overall Appearance, (b) FEM model (c) method to set simulating rotor offset.

Table 2. Setting conditions for FEM simulation

\begin{tabular}{|c|c|c|c|}
\hline Conditions & Rotor offset & Stator deformation & Mixed SAGE \\
\hline Condition 1 & $\delta_{s}=1$ & $\begin{array}{l}n=4, \\
\theta_{1}=0^{\circ}, \theta_{2}=180^{\circ}, \theta_{3}=270^{\circ}, \theta_{4}=90^{\circ} \\
\delta s_{1}=\delta s_{2}=12.5 \%, \delta s_{3}=\delta s_{4}=-12.5 \%\end{array}$ & $\begin{array}{l}\delta_{s}=12.5 \%, n=4, \\
\theta_{1}=0^{\circ}, \theta_{2}=180^{\circ}, \theta_{3}=270^{\circ}, \theta_{4}=90^{\circ} \\
\delta s_{1}=\delta s_{2}=37.5 \%, \delta s_{3}=\delta s_{4}=-37.5 \%\end{array}$ \\
\hline Condition 2 & $\delta_{s}=25 \%$ & $\begin{array}{l}n=4 \\
\theta_{1}=0^{\circ}, \theta_{2}=180^{\circ}, \theta_{3}=270^{\circ}, \theta_{4}=90^{\circ} \\
\delta s_{1}=\delta s_{2}=25 \%, \delta s_{3}=\delta s_{4}=-25 \%\end{array}$ & $\begin{array}{l}\delta_{s}=25 \%, n=4 \\
\theta_{1}=0^{\circ}, \theta_{2}=180^{\circ}, \theta_{3}=270^{\circ}, \theta_{4}=90^{\circ} \\
\delta s_{1}=\delta s_{2}=37.5 \%, \delta s_{3}=\delta s_{4}=-37.5 \%\end{array}$ \\
\hline Condition 3 & $\delta_{s}=37.5 \%$ & $\begin{array}{l}n=4 \\
\theta_{1}=0^{\circ}, \theta_{2}=180^{\circ}, \theta_{3}=270^{\circ}, \theta_{4}=90^{\circ} \\
\delta s_{1}=\delta s_{2}=37.5 \% \delta s_{3}=\delta s_{4}=-37.5 \%\end{array}$ & $\begin{array}{l}\delta_{s}=37.5 \%, n=4 \\
\theta_{1}=0^{\circ}, \theta_{2}=180^{\circ}, \theta_{3}=270^{\circ}, \theta_{4}=90^{\circ} \\
\delta s_{1}=\delta s_{2}=37.5 \% \delta s_{3}=\delta s_{4}=-37.5 \%\end{array}$ \\
\hline
\end{tabular}




\subsection{Results and discussion}

\subsubsection{MPPUA vector diagrams results}

Fig.3 are the MPPUA vector diagrams on stator inner surface under different performing conditions. It shows that the distribution of MPPUA is symmetrical in normal condition, due to the magnetic pole. When the rotor offset occurs, the MPPUA in X direction (also the SAGE direction) becomes larger, while reducing in the opposite direction, as indicated in Fig.3 (a) to (d).. This is consistent with previous theories that the MPPUA is negatively correlated with the size of the air-gap.

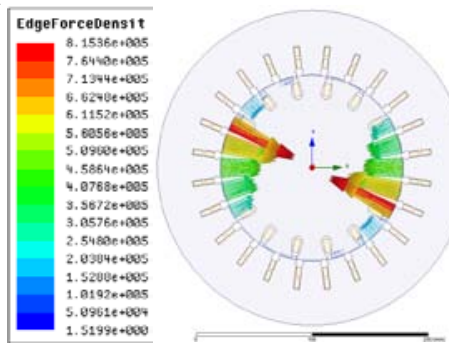

(a) Normal condition

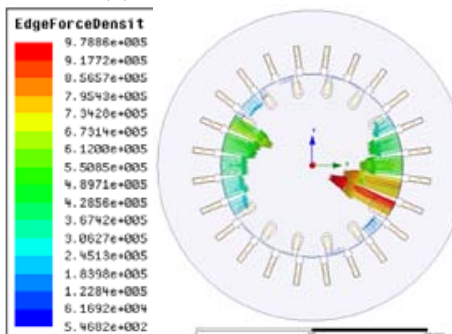

(d) $37.5 \%$ rotor offset

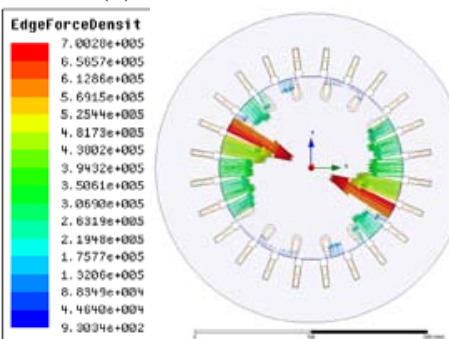

(g) $37.5 \%$ stator deformation

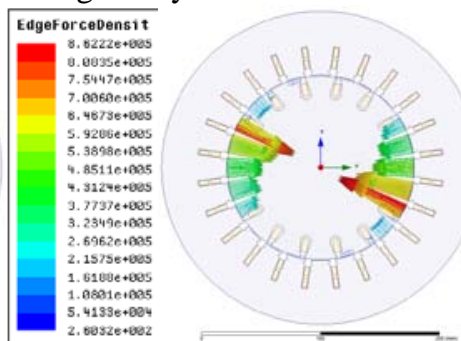

(b) $12.5 \%$ rotor offset

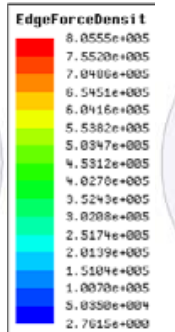

(e) $12.5 \%$ stator deformation

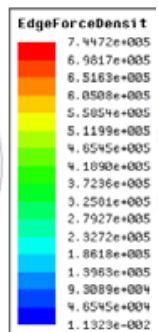

$23 e-002$

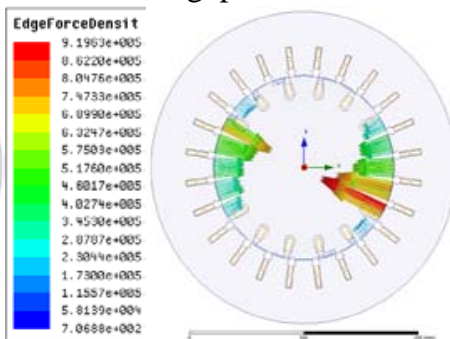

(c) $25 \%$ rotor offset

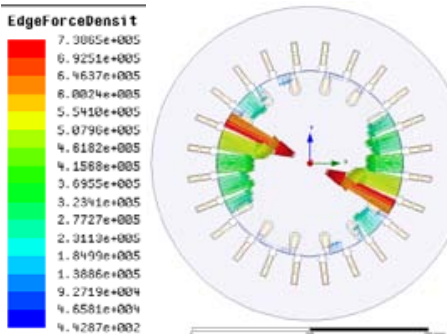

(f) $25 \%$ stator deformation

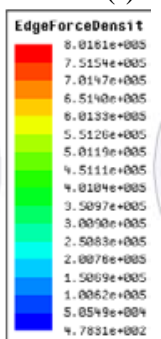

(i) $25 \%$ mixed SAGE

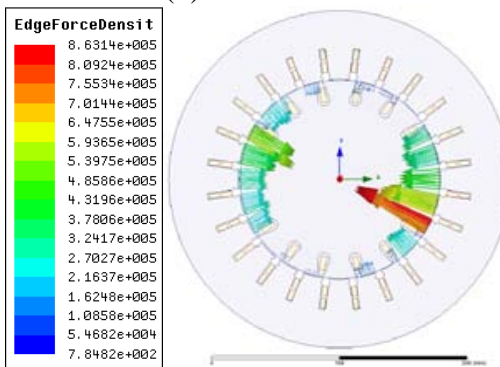

(j) $37.5 \%$ mixed SAGE

Figure 3. Stator MPPUA vector diagrams under different conditions

Besides rotor offset static eccentricity, there is stator overall elliptical deformation fault in generator. Because the ellipse deformation is symmetrical, the distribution of MPPUA is also symmetrical, which can be see in Fig.3 (e) to (g). The color of the arrows are symmetrical, which means the MPPUA is also symmetrical. 
The more common case is that rotor SAGE and stator SAGE exist at the same time. Fig.3 (h) to (j) shows the changes of the distribution of the MPPUA in mixed SAGE. Taking the right part of the vector diagrams for an example, the value of the MPPUA (show in the arrows) decreases at first, when the mixed SAGE occurs. Then the value begins increasing as the SAGE develops. This is mainly because the average length of the air-gap increase first and then decrease.

\subsubsection{MPPUA spectra characteristics results}

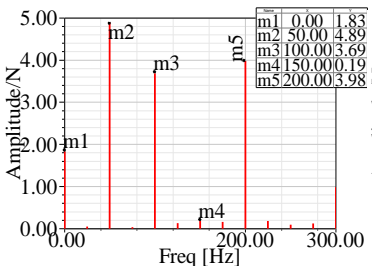

(a) Normal condition

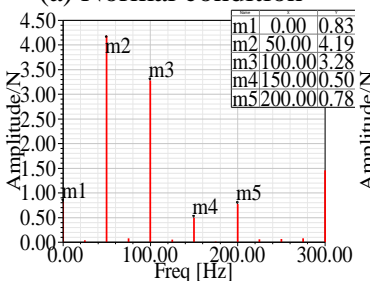

(e) $12.5 \%$ stator deformation

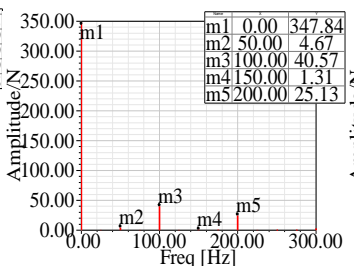

(b) $12.5 \%$ rotor offset

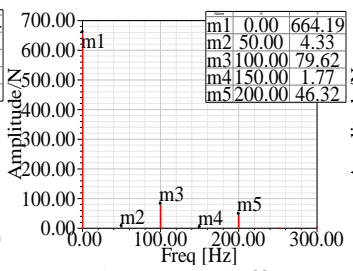

(c) $25 \%$ rotor offset

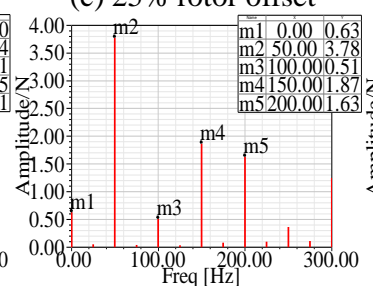

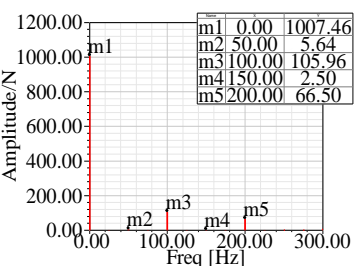

(d) $37.5 \%$ rotor offset
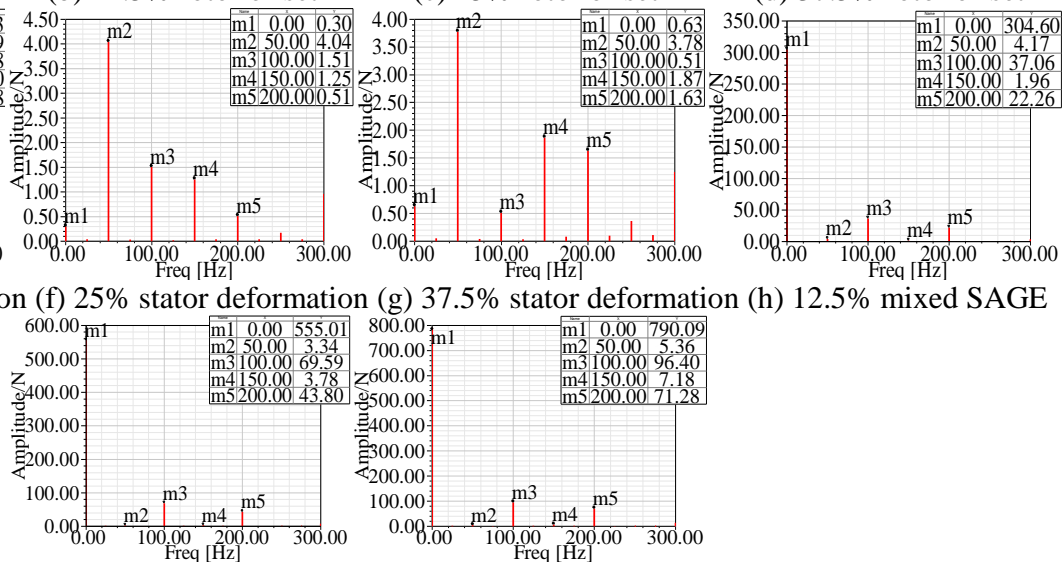

(i) $25 \%$ mixed SAGE

(j) $37.5 \%$ mixed SAGE

Figure 4. Stator MPPUA spectra under different conditions

The stator MPPUA spectra characteristics under different conditions are shown in Fig.4. Both DC component, $100 \mathrm{~Hz}$ and other even harmonic component $(200 \mathrm{~Hz})$ are increased as rotor SAGE develops, as indicated in Fig.4 (a) to (d). Compared with the normal condition, as rotor offset is increased to $12.5 \%$, 25\%, 37.5\%, respectively, the 2nd harmonic of MPPUA increased by $999.46 \%$, $2057.72 \%$, and $2771.54 \%$, while the 4 th harmonic increased by $531.41 \%$, $1063.82 \%$, and $1570.85 \%$. Obviously, the rotor offset can cause the stator vibrate with double times rotating frequency, and as rotor SAGE develops, the MPPUA will be greater and vibrations will be more intensive. Meanwhile due to the higher odd harmonic component of the MFD and the MMF, the even harmonic component will appear by the square operation in Eq.(6). This explains the growth trend of 4th harmonic.

The MPPUA spectra under stator ellipse deformation fault are shown in Fig.4 (e) to (g). Compared with the normal condition, as stator ellipse deformation is increased to $12.5 \%, 25 \%$, 37.5\%, respectively, the 2nd harmonic of MPPUA decreased by $11.11 \%$, 59.08\%, and $86.18 \%$. Theoretically, the vibration amplitudes basically unchanged under stator ellipse deformation, which is true for unchanged average air-gap length situation. The decreasing trend of MPPUA probably because the simulation settings are not extremely accurate, the 4 deformations (see in Fig, 4 (c)) are not equal in size . In modeling, the average air-gap length is increased slightly due to elliptical deformation of stator. So ignoring the small change of the average air-gap length, the vibration amplitudes of MPPUA can be considered basically unchanged.

The MPPUA spectra under mixed SAGE fault are shown in Fig.4 (h) to (j). As we can see from Fig.4 (h) to (j), both DC component, $100 \mathrm{~Hz}$ and other even harmonic component $(200 \mathrm{~Hz}) \mathrm{will}$ be 
increased as mixed SAGE develops. Besides, due to the state of mixed SAGE, the average value of the air-gap length $g_{0}$ in mixed SAGE can be much larger than that in rotor offset case, therefore, the spectra will be less intensive (see in Fig.4 (b)(c)(d) and Fig.4 (h)(i)(j)). The simulation results agree with the theoretical.

The MPPUA amplitudes in different SAGE cases are summarized in Tab.3. From the results above, we can see that the impact on stator MPPUA and vibration characteristics from big to small in turn for: rotor offset, mixed SAGE, stator ellipse deformation. This is mainly caused by the air-gap condition, the SAGE under rotor offset is the biggest change due to the rotor overall displacement. Follow by the SAGE under stator ellipse deformation, because the air-gap deformation is symmetrical, the MPPUA are set off against each other. The SAGE under mixed eccentricity fault is in the middle, because it combines the air-gap characteristic of rotor offset and stator ellipse deformation.

Table 3. MPPUA amplitudes of different SAGE cases

\begin{tabular}{|c|c|c|c|c|}
\hline \multirow{2}{*}{ SAGE cases } & \multicolumn{4}{|c|}{$2 f$ pulsating component amplitudes under different SAGE degree } \\
\cline { 3 - 5 } & normal & $\delta=12.5 \%$ & $\delta=25 \%$ & $\delta=37.5 \%$ \\
\hline \multirow{2}{*}{ Rotor offset } & \multirow{3}{*}{3.69} & 40.57 & 79.62 & 105.96 \\
\cline { 4 - 5 } \cline { 3 - 5 } & & 3.28 & 1.51 & 0.51 \\
\cline { 3 - 5 } Stator ellipse deformation & 37.06 & 69.59 & 96.40 \\
\hline Mixed SAGE & & &
\end{tabular}

\section{Conclusions}

Through theoretical derivation and simulation verification, this paper gets the following conclusion:

(1) Rotor SAGE will cause the stator vibrate with double times rotating frequency. As rotor SAGE develops, the MPPUA will be greater and vibrations will be more intensive.

(2) Stator SAGE cause the stator's vibration is related to the deformation number $n$, the level of deformation $\Lambda_{s n}^{\prime}$, and the angle $\theta_{n}$ between the $n$-th deformation and $\mathrm{X}$ axis. If the deformations are symmetrically distributed in preciseness, the integral MPPUA acting on the stator can be zero. And if the deformations are unsymmetrical, the stator will vibrate with double times rotating frequency. As stator deformation SAGE develops, the MPPUA will be greater and vibrations will be more intensive.

(3) Mixed SAGE can be regarded as the superposition of rotor static eccentricity and stator static eccentricity. It will cause the rotor vibrate with double times rotating frequency. As mixed SAGE develops, the MPPUA will be increased and vibrations will be more intensive.

(4) MPPUA will be increased as the reduction of the air-gap length. The direction of the integral MPPUA will be directed from the smaller side of the air-gap to the larger side.

(5) Simulation result shows that the impact of MPPUA from big to small is rotor offset static eccentricity fault, stator mixed SAGE, and stator core ellipse deformation fault.

These conclusions can provide the basis for fault diagnosis of generator.

\section{Acknowledgement}

This work is supported by the national natural science foundation of China (No.51307058), the Natural Science Foundation of Hebei Province, China (E2014502052, E2015502013), and the Chinese Fundamental Research Funds for the Central Universities (2015ZD27).

\section{References}

1. S.T. Wan, Y.L. He, G.J. Tang, "Analysis on Stator Circulating Current Characteristics under Eccentricity Faults of Turbo-generator", High Voltage Engineering, 36, 6, pp.1547-1553, (2010)

2. S.T. Wan, H.M. Li, Y.G. Li, "Analysis on vibration characteristics of generator with the fault of eccentric air-gap", Journal of Vibration and Shock, 24, 6, pp.21-23, (2005) 
3. Y.L. He, S.T. Wan, G.J. Tang, L. Xiang, "Eccentric fault level identification of a turbo generator based on stator vibration characteristics", Journal of Vibration and Shock, 31, 22, pp.53-57, (2012)

4. D. Ye, "Electrical Machinery", Tianjin Science and Technology Press, pp.147-149, (1995)

5. Sarikhani, O.A. Mohammed, "Inter-Turn Fault Detection in PM Synchronous Machines by Physics-Based Back Electromotive Force Estimation", IEEE Transactions on Industrial Electronics, 60, 8, pp.3472-3484, Aug. (2013)

6. M. Yousefi Kia, M. Khedri, H.R. Najafi, M.A.S. Nejad, "Hybrid modelling of doubly fed induction generators with inter-turn stator fault and its detection method using wavelet analysis", Generation, Transmission \& Distribution, IET , 7, 9, pp.982-990, Sept. (2013)

7. W. Doorsamy, A.A.E. Abdallh, W.A. Cronje, L. Dupré, "An Experimental Design for Static Eccentricity Detection in Synchronous Machines Using a Cramér-Rao Lower Bound Technique", IEEE Transactions on Energy Conversion, 30, 1, pp.254-261, March (2015)

8. Bruzzese, G. Joksimovic, "Harmonic Signatures of Static Eccentricities in the Stator Voltages and in the Rotor Current of No-Load Salient-Pole Synchronous Generators", IEEE Transactions on Industrial Electronics, 58, 5, pp.1606-1624, May (2011) 